\title{
Ionization processes in a local analogue of distant clumpy galaxies: VLT MUSE IFU spectroscopy and FORS deep images of the TDG NGC $5291 N^{\star}$
}

\author{
J. Fensch ${ }^{1}$, P.-A. Duc ${ }^{1}$, P. M. Weilbacher ${ }^{2}$, M. Boquien ${ }^{3,4}$, and E. Zackrisson ${ }^{5}$ \\ ${ }^{1}$ Laboratoire AIM Paris-Saclay, CEA/IRFU/SAp, Université Paris Diderot, 91191 Gif-sur-Yvette Cedex, France \\ e-mail: jeremy.fensch@cea.fr \\ 2 Leibniz-Institut für Astrophysik, An der Sternwarte 16, 14482 Potsdam, Germany \\ 3 Institute of Astronomy, University of Cambridge, Madingley Road, Cambridge, CB3 0HA, UK \\ ${ }^{4}$ Unidad de Astronomía, Fac. de Ciencias Básicas, Universidad de Antofagasta, Avda. U. de Antofagasta 02800, Antofagasta, Chile \\ 5 Department of Physics and Astronomy, Uppsala University, 75120 Uppsala, Sweden
}

Received 7 August 2015 / Accepted 28 September 2015

\section{ABSTRACT}

\begin{abstract}
Context. We present Integral Field Unit (IFU) observations with MUSE and deep imaging with FORS of a dwarf galaxy recently formed within the giant collisional HI ring surrounding NGC 5291. This Tidal Dwarf Galaxy (TDG) -like object has the characteristics of typical $z=1-2$ gas-rich spiral galaxies: a high gas fraction, a rather turbulent clumpy interstellar medium, the absence of an old stellar population, and a moderate metallicity and star formation efficiency.

Aims. The MUSE spectra allow us to determine the physical conditions within the various complex substructures revealed by the deep optical images and to scrutinize the ionization processes at play in this specific medium at unprecedented spatial resolution.

Methods. Starburst age, extinction, and metallicity maps of the TDG and the surrounding regions were determined using the strong emission lines $\mathrm{H} \beta$, [OIII], [OI], [NII], $\mathrm{H} \alpha$, and [SII] combined with empirical diagnostics. Different ionization mechanisms were distinguished using BPT-like diagrams and shock plus photoionization models.

Results. In general, the physical conditions within the star-forming regions are homogeneous, in particular with a uniform half-solar oxygen abundance. On small scales, the derived extinction map shows narrow dust lanes. Regions with atypically strong [OI] emission line immediately surround the TDG. The $[\mathrm{OI}] / \mathrm{H} \alpha$ ratio cannot be easily accounted for by the photoionization by young stars or shock models. At greater distances from the main star-foming clumps, a faint diffuse blue continuum emission is observed, both with the deep FORS images and the MUSE data. It does not have a clear counterpart in the UV regime probed by GALEX. A stacked spectrum towards this region does not exhibit any emission line, excluding faint levels of star formation, or stellar absorption lines that might have revealed the presence of old stars. Several hypotheses are discussed for the origin of these intriguing features.
\end{abstract}

Key words. HII regions - galaxies: dwarf - galaxies: ISM - galaxies: individual: NGC 5291N - galaxies: starburst galaxies: interactions

\section{Introduction}

Under the current paradigm of a $\Lambda$ CDM cosmology, dwarf galaxies are considered the building blocks of today's massive galaxies (Kauffmann et al. 1993), and as such numerous detailed studies are devoted to nearby dwarfs. Among them, the starbursting blue compact dwarf galaxies (BCDGs), which are less than $1 \mathrm{kpc}$ large and show very low metallicities $(1 / 40$ th $Z_{\odot}<Z<Z_{\odot} / 3$ ), have long been believed to be very young objects (Sargent \& Searle 1970; Kunth et al. 1988), just like the newly formed galaxies at high redshift. However, the detection of extended red stellar emission (Loose \& Thuan 1986; Papaderos et al. 1996) have shown that most BCDGs are actually old systems.

The object investigated in this paper, NGC 5291N (NED ${ }^{1}$ distance: $63.1 \mathrm{Mpc}$ ), was originally classified as a BCDG (Maza et al. 1991). It does, however, stand out because it shows

* Based on observations collected at the European Organisation for Astronomical Research in the Southern Hemisphere, Chile: ESO MUSE programme 60.A-9320(A) and FORS programme 382.B0213(A).

1 NASA/IPAC Extragalactic Database. no hint of any old stellar component (Boquien et al. 2007, 2010), and it is very gaseous $-M_{\text {gas }}$ is around $85 \%$ of the total mass (Bournaud et al. 2007) - and has a clumpy structure. Its metallicity, about half-solar (Duc \& Mirabel 1998, hereafter DM98), is higher than in classical BCDGs, but not so different from for a number of distant star-forming galaxies with metallicities greater than $0.4 Z_{\odot}$ at $z=2$ (Erb et al. 2006; Stark et al. 2008; Steidel et al. 2014; Zanella et al. 2015). Therefore, the dynamically young dwarf NGC 5291N (Bournaud et al. 2007) appears as one of the most promising (low-mass) analogues of the distant gas-dominated clumpy galaxies, which are found to be numerous in deep cosmological fields. It was formed in a huge gaseous ring $\left(M_{\mathrm{HI}}>10^{10} M_{\odot}\right.$, DM98) surrounding the early-type galaxy NGC 5291, which expanded in the intergalactic medium after a violent collision with a bullet galaxy. According to numerical simulations, the encounter occurred 360 million years ago (Bournaud et al. 2007). The HI rich ring formed a series of starforming clumps. A kinematical analysis (Bournaud et al. 2007; Lelli et al. 2015) revealed that three of the gas condensations are most likely gravitationally bound. Our target, NGC 5291N, is the most massive of them. Similar to tidal dwarf galaxies (TDGs, see review by Duc \& Mirabel 1999), NGC 5291N has low dark 
matter (DM) content. As a matter of fact, NGC 5291N shares many properties with the $10^{8} M_{\odot}$ individual clumps, which formed within the gravitationally unstable gas-dominated disk of distant clumpy galaxies (Zanella et al. 2015), and they are also presumably dark-matter poor. The stability of such objects against internal feedback is a matter of active debate (e.g. see Genel et al. 2012; Bournaud et al. 2014). It can be investigated in NGC $5291 \mathrm{~N}$ with great accuracy, keeping in mind, however, that the star-formation rate of this local analogue is much lower: around $0.14 M_{\odot} \mathrm{yr}^{-1}$ for NGSC 5291N (Boquien et al. 2010) compared to $32 \pm 6 M_{\odot} \mathrm{yr}^{-1}$ for the $z=2$ clump in Zanella et al. (2015).

The ring of NGC 5291 and its dwarf galaxies within it have been observed by a wide range of ground-based and spatial instruments, resulting in an extensive wavelength coverage: HI 21$\mathrm{cm}$ line with the VLA (Bournaud et al. 2007), far-infrared with PACS and SPIRE on Herschel (Boquien et al., in prep.), midinfrared with Spitzer (Boquien et al. 2007), near-infrared with ISAAC on the ESO VLT, optical slit with EMMI on the NTT, and $\mathrm{H} \alpha$ integral field spectroscopy with a Fabry-Perot (FP) unit on the ESO $3.6 \mathrm{~m}$ (Bournaud et al. 2004), and far and near ultraviolet with GALEX (Boquien et al. 2007). The slit optical spectroscopy of NGC 5291N disclosed a number of bright emission lines, presumably associated with H II regions, and FP observations revealed complex kinematical features within the dwarf on top of the ordered rotation also seen in the HI component.

A better understanding of the physical conditions in the interstellar medium (ISM) requires 3D spectroscopic information at good spatial and spectral resolution and a relatively large field of view. This is the capability of the Multi Unit Spectroscopic Explorer (MUSE), which has been recently mounted on the VLT at Paranal observatory (Bacon et al. 2010) and which is about to revolutionize this field of research. We present here MUSE observations of NGC 5291N. They provide us with several tens of thousand resolved spectra and allow us to probe the ionizing processes in the dwarf on physical spatial scales of only $200 \mathrm{pc}$. Their exploitation is backed by deep multi-band images obtained with the FORS instrument on the ESO VLT.

Former IFU observations of dwarf galaxies have already given us some insight into the ionization properties of these galaxies (Izotov et al. 2006; Lagos et al. 2009, 2012, 2014; James et al. 2009, 2010, 2013a,b) and have led to identifying (i) regions with Wolf-Rayet stars (Cairós et al. 2010; Kehrig et al. 2013); (ii) the presence of non-thermal ionization processes such as shocks (Cairós et al. 2010); or (iii) the existence of a central AGN (Cairós et al. 2009). To our knowledge, this paper is only the second one presenting MUSE observations of a dwarf galaxy and the first one focused on a colliding system. Previously, ionized cones associated with formerly observed Ly $\alpha$ photon leakage, and possibly correlated with outflows, have been identified around the starburst ESO 338-IG04 (Bik et al. 2015).

The paper is structured as follows. Section 2 describes the observation and data reduction procedures. Section 3 presents the detailed analysis of the emission lines and continuum emission, with a focus on their spatial variations. These results are discussed in Sect. 4, and the final conclusions are drawn in Sect. 5.

\section{Observation and data reduction}

\subsection{MUSE data}

NGC 5291N was observed using the integral field spectrograph MUSE (Bacon et al. 2010) mounted on the Very Large Telescope (VLT). The observations were performed on June 2014 during the first science verification run (60.A-9320(A), PI: P.-A. Duc). We obtained spectra between $4750 \AA$ and $9350 \AA$ over a field of view of $1^{\prime} \times 1^{\prime}$, which corresponds to $18 \mathrm{kpc} \times 18 \mathrm{kpc}$ at a distance of $63.1 \mathrm{Mpc}$.

The observations consisted of three on source exposures of $600 \mathrm{~s}$ each, obtained at three position angles $\left(0,90\right.$, and $\left.180^{\circ}\right)$. Two exposures of $100 \mathrm{~s}$ were done on an offset (blank) sky field. Twilight sky flats taken the following evening were used in the data reduction process, and an exposure of the spectrophotometric standard star LTT 7987 taken in the following morning twilight was used for flux calibration. The reduction used the MUSE pipeline (Weilbacher et al. 2012) through the ESOREX programme. We used a development version of the pipeline, but the code was very close to the 1.0 release $^{2}$.

We followed the usual steps, with bias subtraction, flatfielding, and spectral tracing using the lamp-flat exposures and wavelength calibration, all using daytime calibrations made the morning after the observations. The standard geometry table and astrometric solution derived during Commissioning $2 \mathrm{a}$ were used to create the intermediate pixel tables. Since the observations were done at a low ambient temperature of $\sim 6^{\circ} \mathrm{C}$, slice 6 of the channel was not illuminated and could not be traced. We followed the procedure outlined in the pipeline manual to delete the data of this slice from the science pixel tables.

Sky spectra were created using $75 \%$ of the field of view of the two offset sky fields, and the line-spread function derived from the same wavelength calibration exposures was then used to decompose the spectrum into sky emission lines and continuum.

The science reduction was then assigned the sky continuum that was taken closest in time to the science exposure, and we allowed the sky emission line fluxes to be re-adjusted for the science data. The data were corrected for atmospheric refraction and to barycentric velocities (the corrections were about $-23.8 \mathrm{~km} \mathrm{~s}^{-1}$ ), before being combined into the final cube. We chose a non-standard sampling in the spectral direction, so that the output cube has steps of $0.2 \times 0.2 \times 0.8 \AA$. The spatial FWHM measured on the white-light image created from this final, combined cube is about $0 .{ }^{\prime} 8$.

An example of the extracted spectrum for a single spaxel is shown in Fig. 1. It exhibits the very strong emission lines of the Balmer lines $\mathrm{H} \beta$ and $\mathrm{H} \alpha$, but also forbidden lines such as [OIII] $\lambda \lambda 4959,5007,[\mathrm{NII}] \lambda 6583,[\mathrm{SII}]^{3} \lambda \lambda 6717,6731$, and [OI] 26300 . To create reliable spatial maps of line fluxes, the data cube was re-binned by $3 \times 3$. Given the depth of the data, using an adaptative smoothing routine was not necessary: for most regions, a signal-to-noise ratio $(\mathrm{S} / \mathrm{N})$ higher than 3 was reached with the adopted fixed $3 \times 3$ binning. The resulting spatial dimensions of the final $3 \times 3$ spaxels are $0 .{ }^{\prime} 6 \times 0.0^{\prime} 6$ or $180 \mathrm{pc} \times 180 \mathrm{pc}$.

The continuum emission was extracted from the spectra with the PYRAF continuum procedure. Emission line fluxes were computed with custom-made Python scripts based on Gaussian fits. We only studied regions where the emission was $3 \sigma$ above the noise, and we made sure that our Gaussian fitting error was less than $10 \%$ of the total line flux. Unfortunately, the redshift of the galaxy put the $[\mathrm{SII}]_{\lambda 6731}$ very close to airglow lines at $6827 \AA$, making it very hard to detect at low luminosity. However, the $[\mathrm{SII}] \lambda 6717 /[\mathrm{SII}] \lambda 6731$ ratio is a tracer of the electron density. In the low-density limit, it reaches 1.45 . In the brightest region in

\footnotetext{
2 Available from ESO via http://www.eso.org/sci/software/ pipelines/muse/muse-pipe-recipes.html

3 Unless stated otherwise, $[\mathrm{SII}]$ refers to $[\mathrm{SII}] \lambda 6717+[\mathrm{SII}] \lambda 6731$.
} 

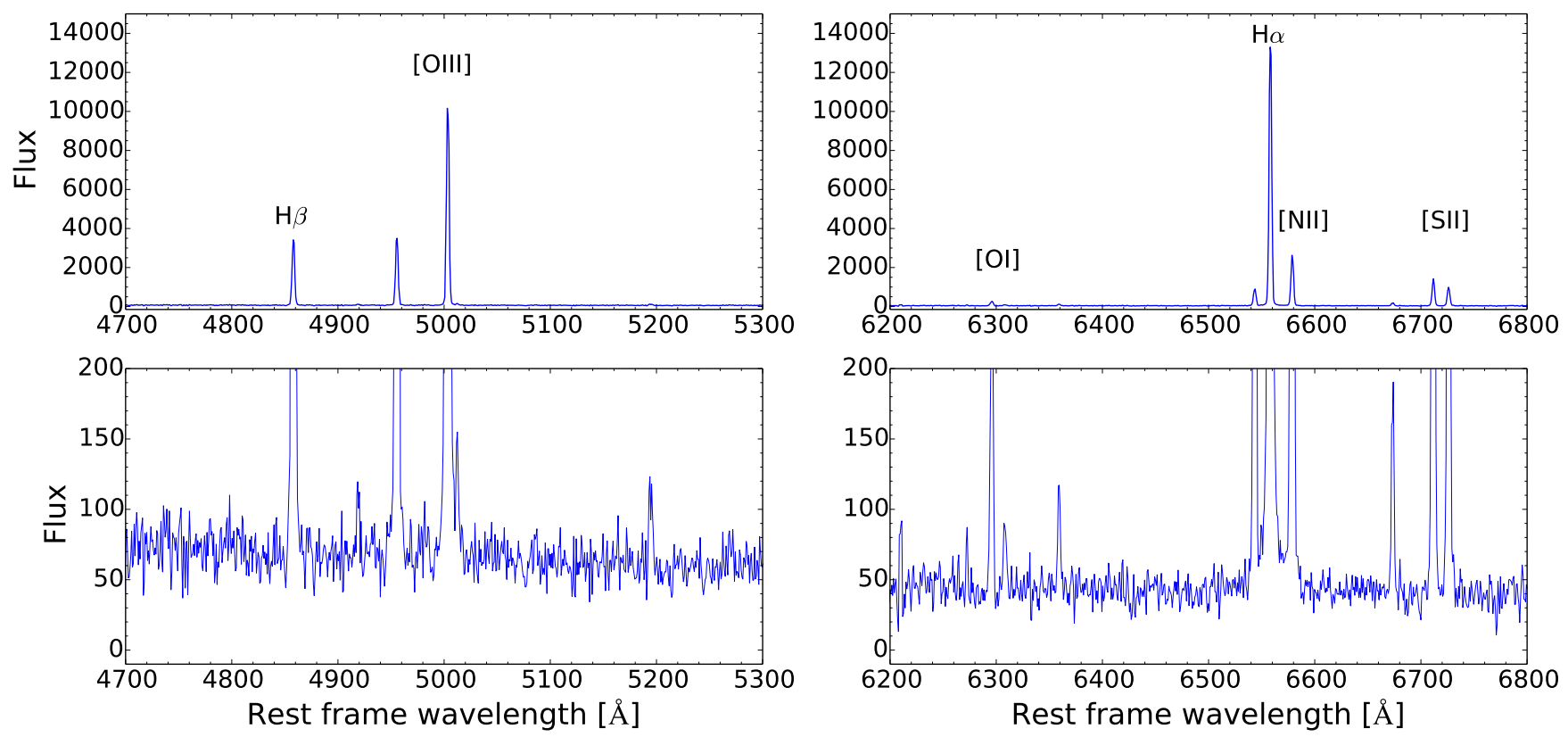

Fig. 1. Top: spectrum of a single spaxel, before the re-binning, at the position of the brightest region with the position of the main emission lines. Bottom: zoom on the same spectrum. The flux unit is $10^{-20} \mathrm{erg} \mathrm{cm}^{-2} \mathrm{~s}^{-1}$.

our field, the lowest value is 1.15 , corresponding to an electron density of $300 \mathrm{~cm}^{-3}$. Therefore, in the case of a non-detection of $[\mathrm{SII}]_{\lambda 6731}$ but a good detection of every other lines, a value of $[\mathrm{SII}]_{\lambda 6731}=[\mathrm{SII}]_{\lambda 6717} / 1.42$ is chosen, which corresponds to a maximum error of $9 \%$ on the value of $[\mathrm{SII}]=[\mathrm{SII}]_{\lambda 6717}+$ $[\mathrm{SII}]_{\lambda 6731}$ for the densest regions, which is lower than the uncertainty on the Gaussian fit and less than $2 \%$ for regions with $n_{\mathrm{e}}<100 \mathrm{~cm}^{-3}$, which are the hardest to resolve. The uncertainty for the value of $[\mathrm{SII}]_{\lambda 6731}$ is not available for the spaxels that need this method, because it is dominated by the noise from the sky line. When mean uncertainties involving the [SII] emission doublet are to be assessed, points where $[\mathrm{SII}]_{\lambda 6731}$ is blended by the airglow line will not be taken into account.

\subsection{FORS data}

The deep optical $V, R$, and $I$ images were acquired in March 2010 with the instrument FORS on the VLT (Programme 382.B0213(A), PI: E. Zackrisson). The final images, covering a total field of view of $11.2^{\prime} \times 15.5^{\prime}$, were obtained by registering and combining 26 individual exposures of $300 \mathrm{~s}, 300 \mathrm{~s}$, and $240 \mathrm{~s}$ in the $V, R$, and $I$ Bessel filters, respectively. Photometric zero points were provided by the ESO pipeline. The limiting surface brightness in the $V$ band is estimated to $27 \mathrm{mag} \operatorname{arcsec}^{-2}$ (Vega) from the local fluctuations in the background. The average FWHM of point sources in the stacked image is $1.0^{\prime \prime}$.

The FORS image of the whole system, including the collisional ring and its host galaxy, is shown in the left-hand part of Fig. 2, with the HI emission from the VLA superimposed in blue. The radio data highlights the shape of the ring structure. The field of view of the MUSE observations towards the TDG NGC $5291 \mathrm{~N}$ is delineated by the white square. Both the composite FORS broad-band image (Fig. 2, top right) and the MUSE narrow-band image (Fig. 2, bottom right) reveal the clumpy structure of the ISM of NGC 5291N. The FORS image mostly exhibits continuum emission; the MUSE image is a combination of emission from three main lines: [OIII] ( $\lambda 5007), \mathrm{H} \alpha$. and [NII] (26583). As shown by comparing the FORS and MUSE images (see also Fig. 2), the individual clumps are associated to strong emission lines and are embedded in regions with extended diffuse continuum emission.

\section{Results}

As illustrated in Fig. 2, the MUSE spectrophotometric data show a strikingly large heterogeneity towards a recently born object, NGC 5291N, which could a priori be considered simple. While the continuum emission has a rather uniform blue colour, the emission line ratios vary strongly on small scales. We analyse in the following the spatial variations of the observed spectral lines and investigate various diagnostics to account for them.

\subsection{Dust extinction}

Before investigating the spatial variations of the emission line ratios, dust extinction maps were computed to allow for reddening correction of the emission line fluxes. These maps were derived from the $\mathrm{H} \alpha / \mathrm{H} \beta$ Balmer line ratio. The LMC extinction law from Gordon et al. (2003), with $R_{V}=3.41$, was used in combination with a theoretical value for the unobscured line ratio for case B recombination of $\mathrm{H} \alpha / \mathrm{H} \beta=2.86$, for $T_{\mathrm{e}}=10000 \mathrm{~K}$, and $n_{\mathrm{e}}=100 \mathrm{~cm}^{-3}$ (Osterbrock \& Bochkarev 1989).

As shown in Fig. 3, the extinction is globally low but with local variations of Av by up to $1 \mathrm{mag}$. The dust reddening peaks at a value of $A_{V} \simeq 1.25$ mag towards regions where dust emission had been observed with Spitzer and Herschel. The FORS colour image also shows narrow dust lanes at this location (Fig. 4). The extinction derived from the MUSE data is consistent with the one derived by Boquien et al. (2010) from their fit of the spectral energy distribution (SED) probed by the $F U V, N U V, B, V, R, J, H$, and $K$ bands. It is higher than what was originally estimated by DM98 from slit spectroscopy.

In the following, all emission line fluxes have been corrected for dust extinction.

\subsection{Spatial variation of the emission line ratios}

The spatial variations of the main emission line ratios are plotted in Fig. 5. An inside-out positive gradient is seen in 

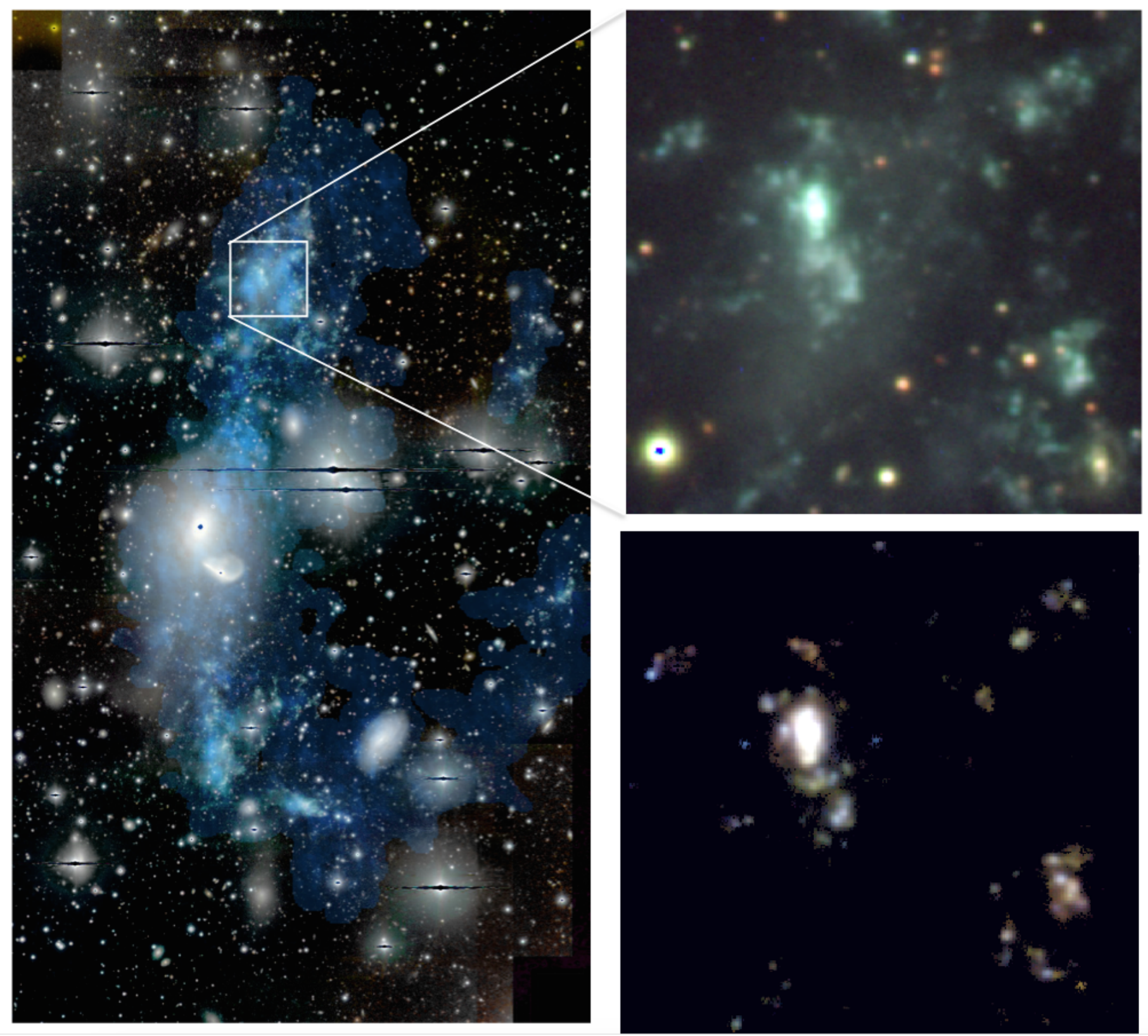

Fig. 2. Left: composite image of the NGC 5291 collisional ring. Background is a FORS composite colour image obtained with the $V, R$, and $I$ bands HI data from the VLA is superimposed in blue. North is up and east left. The white square centred on NGC 5291N delineates the MUSE field of view. Upper right: FORS $V, R, I$ composite colour image of the MUSE target. Lower right: MUSE composite colour image using three different emission lines: Red: [NII] $\lambda 6583$, Green: $\mathrm{H} \alpha$, Blue: [OIII] $\lambda 5007$. Each pixel covers a $60 \mathrm{pc} \times 60 \mathrm{pc}$ region, and the full image corresponds to $1^{\prime} \times 1^{\prime}$, or about $18 \mathrm{kpc} \times 18 \mathrm{kpc}$ at the distance of NGC $5291 \mathrm{~N}, 63.1 \mathrm{Mpc}$.

the $[\mathrm{NII}] \lambda 6584 / \mathrm{H} \alpha,[\mathrm{SII}] \lambda \lambda 6717,6731 / \mathrm{H} \alpha$, and $[\mathrm{OI}] \lambda 6300 / \mathrm{H} \alpha$ maps, whereas the gradient is negative for the $[\mathrm{OIII}] \lambda 5007 / \mathrm{H} \beta$ line ratio. Besides these large-scale variations, some clumps exhibit deviant emission line ratios (see also the lower right panel of Fig. 2). The one immediately north of the TDG has the strongest $[\mathrm{NII}] / \mathrm{H} \alpha$ ratio in the field and the clump to the northwest the strongest $[\mathrm{OIII}] / \mathrm{H} \beta$ ratio.

The flux ratios also depend on the flux intensity of the lines. In Fig. 6, the emission line ratios are plotted as a function of the $\mathrm{H} \alpha$ flux. [SII]/H $\alpha$ and $[\mathrm{OI}] / \mathrm{H} \alpha$ ratios increase significantly with lower $\mathrm{H} \alpha$ surface brightness, whereas [OIII] / $\mathrm{H} \beta$ decreases.

The scatter is large at low $\mathrm{H} \alpha$ surface brightness, partly due to the increase in the measurement errors.
The ISM can be ionized by several mechanisms: photoionization due to ultraviolet (UV) photons from young, hot stars, photoionization from an active galactic nucleus (AGN), shock ionization due to stellar winds and supernovae, or shock ionization from dynamical processes such as collisions. To gain insight into the processes at play, line ratio diagnostics diagrams, known as BPT diagrams (Baldwin et al. 1981; Veilleux \& Osterbrock 1987), are used: [OIII] $\lambda 5007 / \mathrm{H} \beta$ versus $[\mathrm{NII}] \lambda 6584 / \mathrm{H} \alpha,[\mathrm{SII}] \lambda \lambda 6717,6731 / \mathrm{H} \alpha$, and [OI] $\lambda 6300 / \mathrm{H} \alpha$. Although BPT-like diagrams were originally applied to central galactic regions or even to entire galaxies to diagnose AGN or LINER emission, they are used here at the scale of a spaxel to identify the different ionizing processes. 


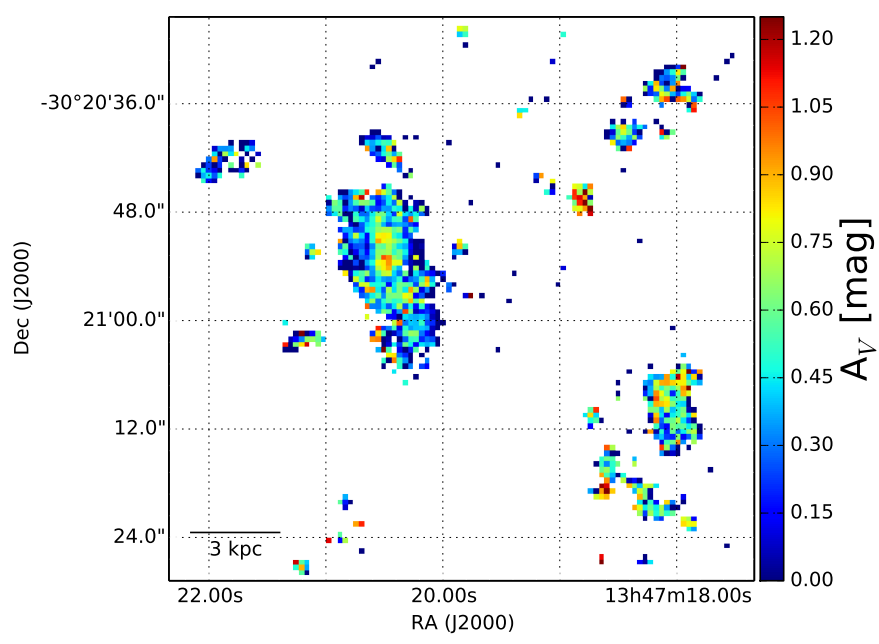

Fig. 3. Spatial distribution of the dust extinction, $A_{V}$. The dust is heterogeneously distributed in the system, but its distribution peaks where star formation is the most recent, see Fig. 8.

Emission line ratios for individual rebinned spaxels are plotted on the BPT diagnostic diagrams shown in Fig. 7. Most of our data points fall in the locus of star-forming regions for two diagnostic diagrams. However, the distribution on the $[\mathrm{OI}] / \mathrm{H} \alpha$ diagnostic does not look standard. It shows a large number of points located outside of the locus for classical H II regions. As shown in the bottom of Fig. 7, the deviant points are located in the outskirts of the star-forming regions. The mean uncertainty on the value of $\log ([\mathrm{OI}] / \mathrm{H} \alpha)$ for the red points is 0.19 , which is not enough to explain their location in the AGN part of the BPT diagram. One should also note that the stacked spectrum of these regions is clearly located in the AGN region of the diagram with very little uncertainty on the measurement.

In the following we investigate the possible causes of the spatial variations of the emission line ratios, starting from the innermost regions to the external ones.

\subsection{Star-forming regions}

The equivalent width of $\mathrm{H} \beta(\mathrm{EW}(\mathrm{H} \beta))$ gives constraints on the age of a starburst episode (Stasińska \& Leitherer 1996; Zackrisson et al. 2001; Terlevich et al. 2004). The spatial distribution shown in Fig. 8 peaks at $230 \AA$ in the centre of the brightest region. $\operatorname{EW}(\mathrm{H} \beta)$ decreases further out to $30 \AA$ in the outskirts of the dwarf. Following Boquien et al. (2007), PEGASE II models (Fioc \& Rocca-Volmerange 1997, 1999) were used to estimate the ages of the star-formation episodes. Assuming an instantaneous starburst and a constant metallicity $Z=0.008$ (see Sect. 3.4), we obtained starburst ages of $3 \mathrm{Myr}$ for the central region and $7 \mathrm{Myr}$ for the oldest regions. Under the assumption that the underlying continuum is only due to the newly formed stars (see Sects. 3.6 and 4.2), the $\operatorname{EW}(\mathrm{H} \beta)$ map looks consistent with an outside-in star-formation episode.

Given the very young age of the central starburst, the presence of Wolf-Rayet (WR) stars is expected. Indeed, DM98 detected a WR bump in their long-slit spectra of NGC 5291N. However, our spectral window does not allow us to reach the location of the bump: the blue boundary of our spectral range is $4682 \AA$ in rest-frame wavelengths, whereas the WR bump peaks at $4686 \AA$. A rising at the far blue end of the spectrum was glimpsed, consistent with the WR feature, but could not be distinguished from an observational bias. Furthermore, the red

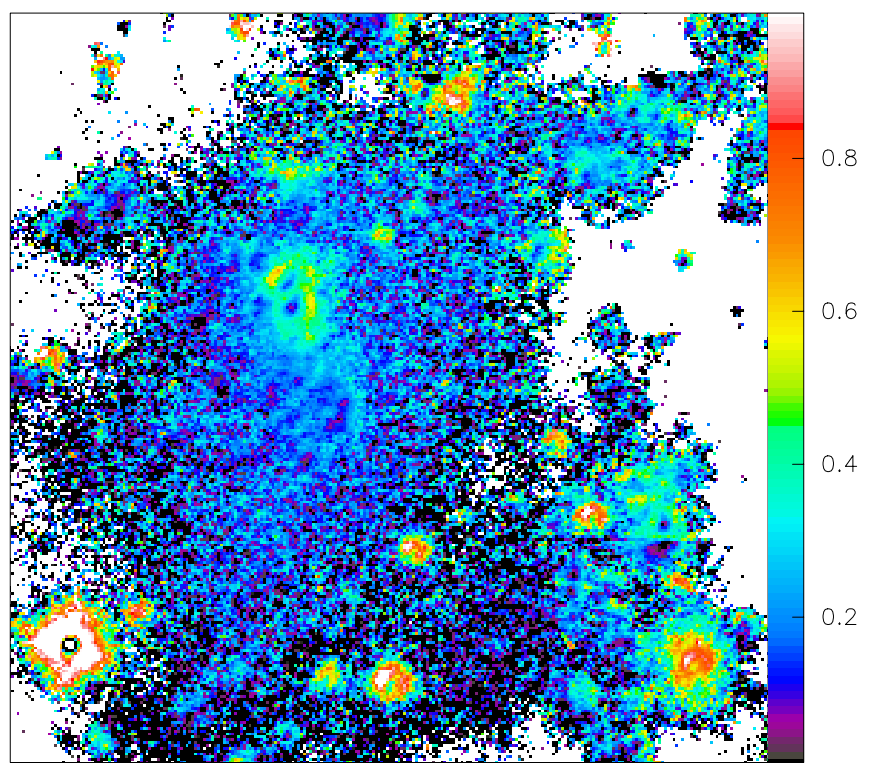

Fig. 4. $V-R$ colour map of NGC 5291N derived from the FORS deep image, scaled in mag. The field of view is the same as in Fig. 3.

WR bump around 5650 to $5800 \AA$ is not observed in the MUSE data.

\subsection{Metallicity}

The chemo-dynamical simulations of Ploeckinger et al. (2014) predict some level of self-enrichment in tidal dwarf galaxies, which should translate into spatial variations of the metallicity and thus contributes to the scatter observed in each BPT diagram of Fig. 7.

We derived the metallicity from the strong emission lines ratios. Unfortunately, our spectral range does not include the [OIII] $\lambda 4363$, so we could not compute the electron temperature, needed for a direct measurement of the metallicity. Instead, we estimated the metallicity from the empirical calibrations based on the parameter $\mathrm{O} 3 \mathrm{~N} 2=\log _{10}\left(\frac{[\mathrm{OIII}] \lambda 5007 / \mathrm{H} \beta}{[\mathrm{NII}] \lambda 6548 / \mathrm{H} \alpha}\right)$. Initial estimates by DM98 indicated rather high values - about half solar - for the oxygen abundance. The Marino et al. (2013) empirical calibration was then chosen. Indeed, compared to other calibrations using this parameter (e.g. Pettini \& Pagel 2004), it includes highmetallicity H II regions and should provide better estimates for high $Z$ regions. Following their calibration of

$12+\log _{10}(\mathrm{O} / \mathrm{H})=8.55-0.221 \times \mathrm{O} 3 \mathrm{~N} 2$

for $\mathrm{O} 3 \mathrm{~N} 2<1.7$, we get $\langle 12+\log (\mathrm{O} / \mathrm{H})\rangle=8.38$ with a standard deviation $\sigma=0.05$, which corresponds to an abundance of $Z \simeq 0.5 Z_{\odot}$, using $12+\log (\mathrm{O} / \mathrm{H})_{\odot}=8.69$ (Asplund et al. 2009). This calibration has a typical uncertainty of $\pm 0.2 \mathrm{dex}$.

The spatial distribution of the metallicity is shown in Fig. 9. An apparent decrease in the metallicity seems to be observed towards the brightest and dustier regions. This is at odds with the simulations of Ploeckinger et al. (2014) that predict a higher metallicity where star formation is the most active.

However, our positive metallicity gradient is $0.2 \mathrm{dex}$, so within the uncertainty of the method we used. It may in fact correspond to variations in other physical parameters, such as the ionization parameter, and the data are consistent with a homogenous metal distribution within NGC 5291N. One should 

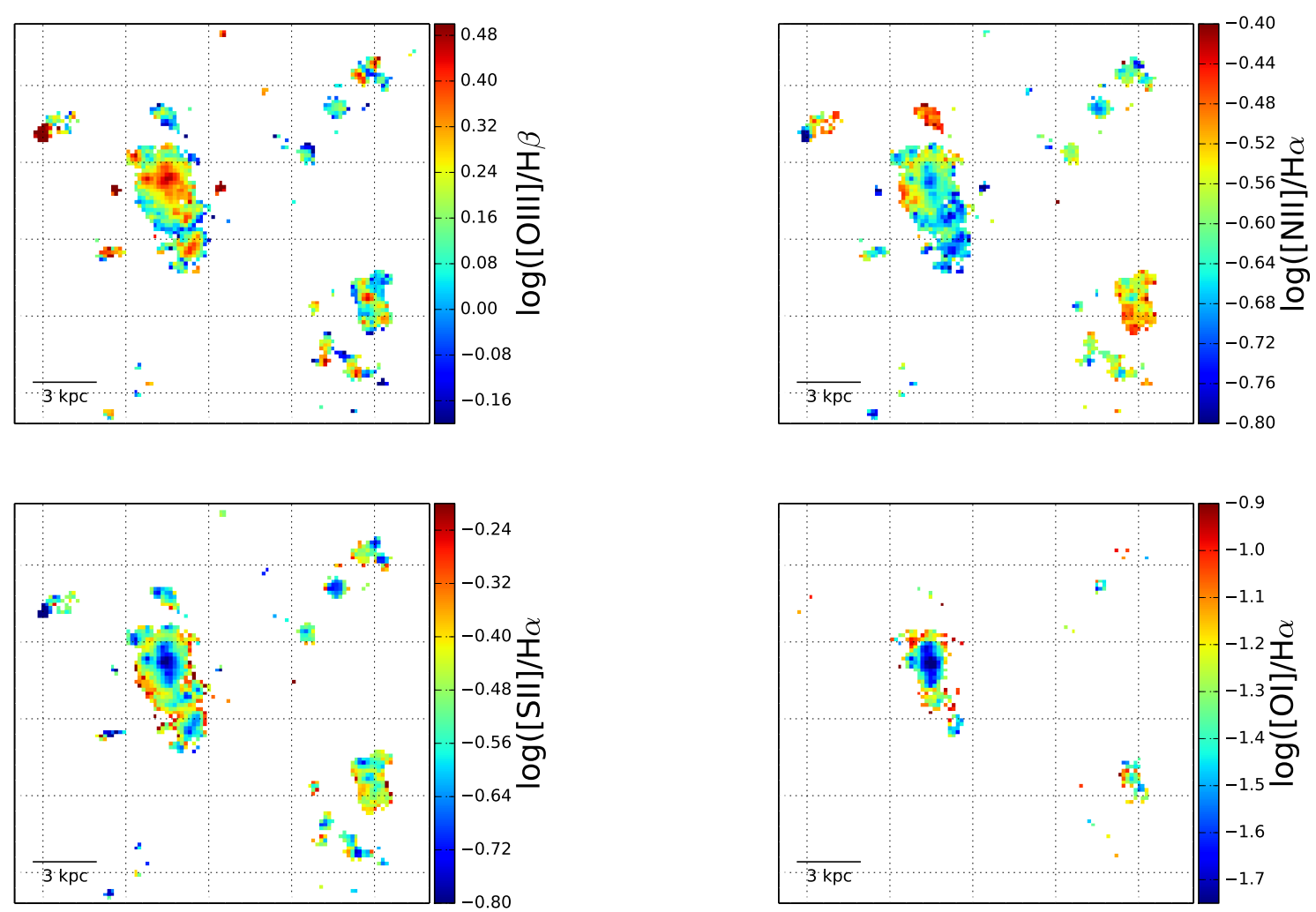

Fig. 5. Spatial distribution of the principal emission-line flux ratios. A logarithmic scale is used for coding the line ratios. The field of view is the same as in Fig. 3.
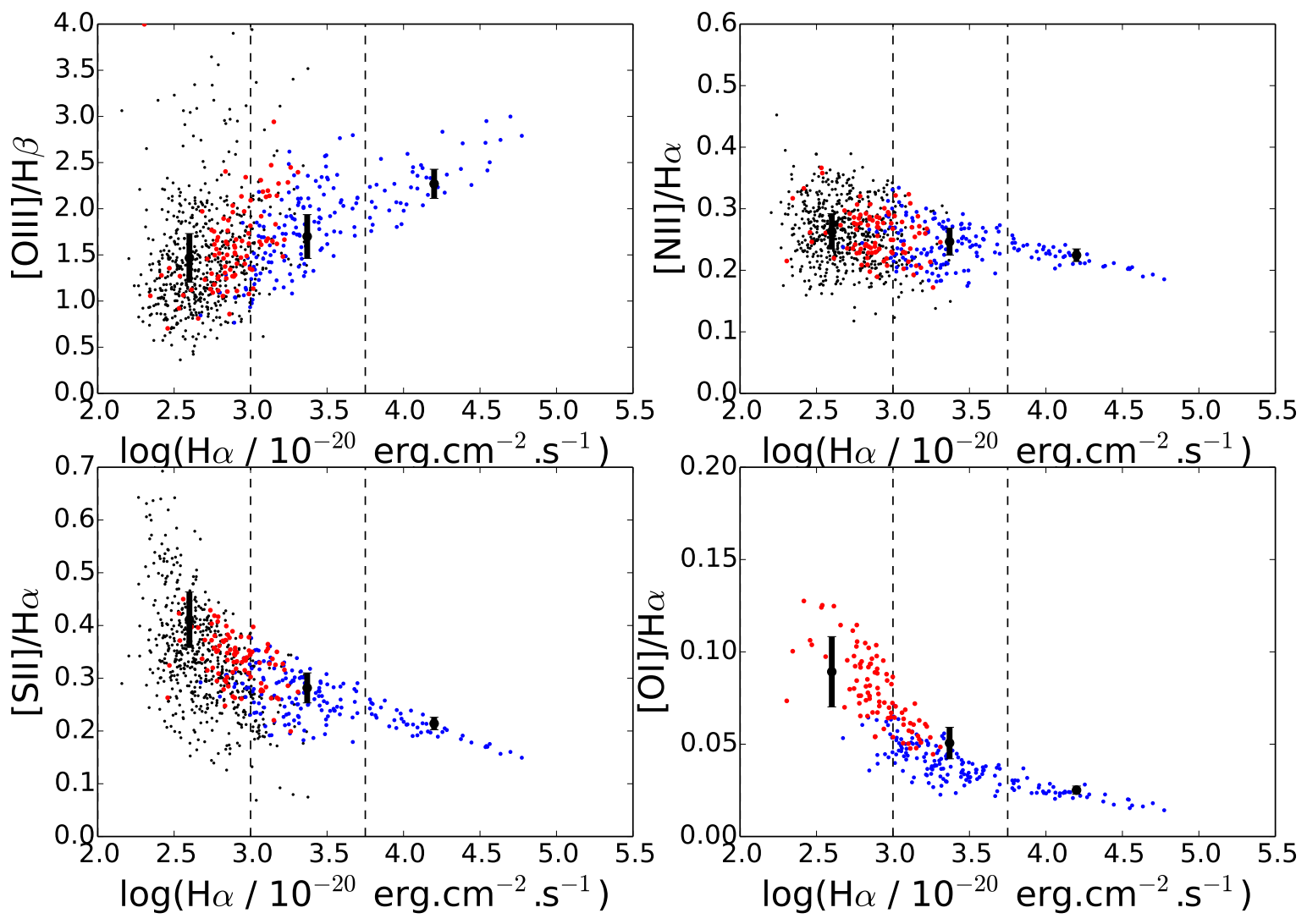

Fig. 6. Distribution of emission-line ratios as a function of the $\mathrm{H} \alpha$ flux, also tracing the local $\mathrm{H} \alpha$ flux surface brightness. The blue, resp. red, points correspond to those located inside, resp. outside, the starburst locus in the [OI]/H $\alpha$ BPT diagnostic diagram (see Fig. 7). The small black points correspond to spaxels with an undetected [OI] line. The error bars are $1 \sigma$ typical dispersion, and their $y$-axis position is the mean value of the ratio in the given $\mathrm{H} \alpha$ flux range. 
J. Fensch et al.: ISM properties of a local analogue to high redshift star-forming clumps
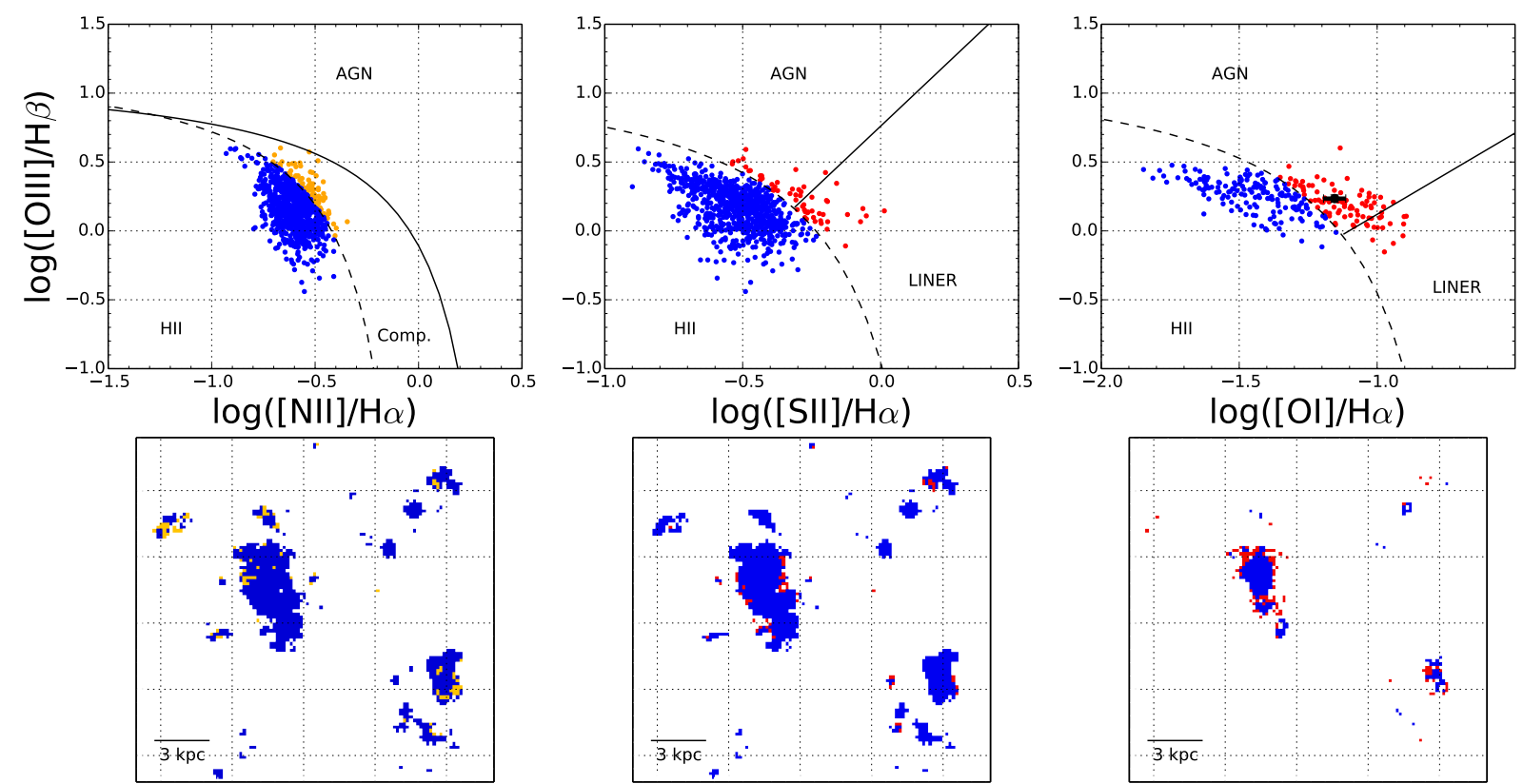

Fig. 7. BPT diagnostics for different emission line ratios. On the $[\mathrm{OIII}] / \mathrm{H} \beta$ vs. $[\mathrm{NII}] / \mathrm{H} \alpha$ diagram, the dotted line delineates the pure starburst region, as defined by Kauffmann et al. (2003) using a set of Sloan Digital Sky Survey (SDSS) spectra. The spaxels belonging to this region are shown in blue, those outside it in orange. The solid line traces the upper theoretical limit to pure H II regions measured by Kewley et al. (2001). For the other diagrams, the dashed lines defined by Kewley et al. (2006) separate the starburst (blue points) from the AGN/Liner (red points) regions. The solid lines further distinguish between AGN ionization (above the line) and LINER ionization (below). The corresponding spatial distribution of starburst/non-starburst $3 \times 3$ binned spaxels are shown on the corresponding plots of the lower panel. The field of view of the plots of the lower panel is the same as in Fig. 3. The highest fraction of spaxels inconsistent with a photoionization by a starburst show up on the $[\mathrm{OIII}] / \mathrm{H} \beta$ vs. $[\mathrm{OI}] / \mathrm{H} \alpha$ diagram. Their spectra have been stacked, and the resulting extracted line ratios are shown with the black point, together with the error bar.

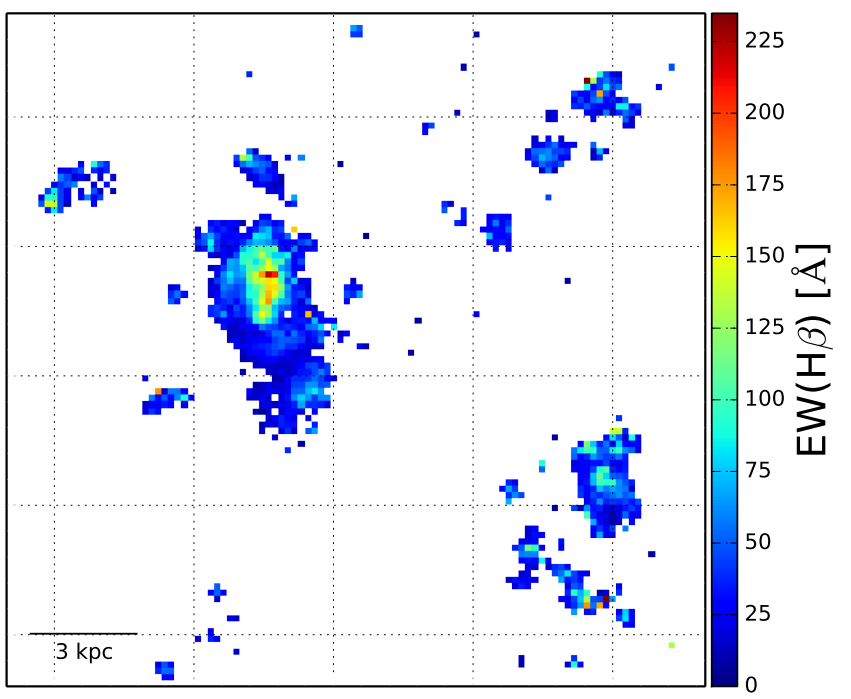

Fig. 8. Spatial distribution of the equivalent width of $\mathrm{H} \beta$. The field of view is the same as in Fig. 3.

note that previous studies, such as DM98, detected no metallicity gradient over the whole HI ring surrounding NGC 5291, whose radius is around $200 \mathrm{kpc}$ and which contains several other star-forming condensations.

\subsection{Ionization parameter}

The ionization parameter $q^{4}$, defined as the ratio between the flux of ionizing photons and the number density of hydrogen

\footnotetext{
4 The dimensionless ionizing parameter $U=q / c$, where $c$ is the speed of light, is used in the following.
}

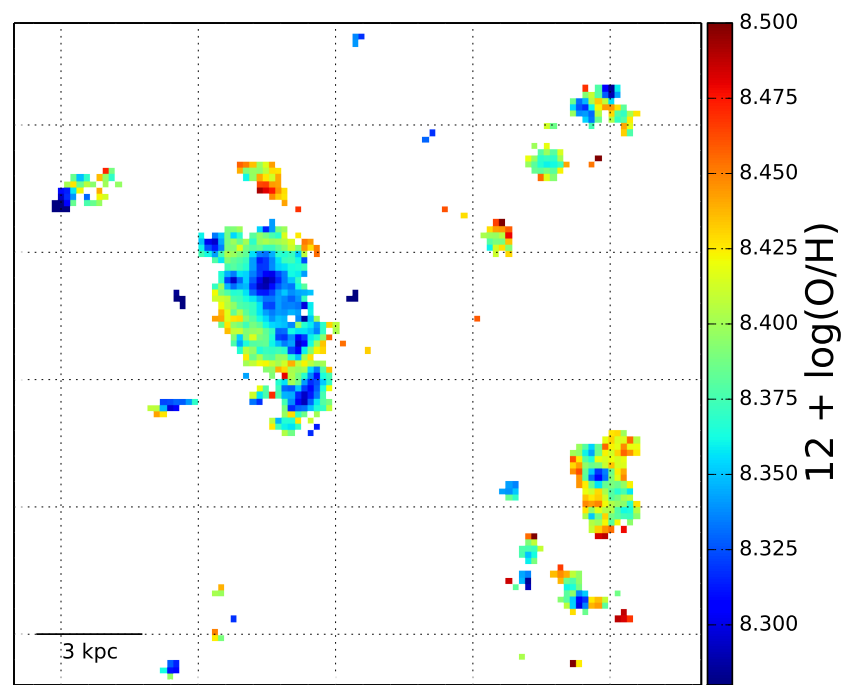

Fig. 9. Metallicity map estimated from $\mathrm{O} 3 \mathrm{~N} 2$ and the calibration of Marino et al. (2013). An outside-in gradient is observed, but the difference between the lowest and the highest abundance can be consistent with an homogeneous abundance distribution and be caused by the variation in other parameters, such as ionization. The field of view is the same as in Fig. 3.

atoms, also has an influence on the values of the emission line ratios (Dopita et al. 2013). To determine the distribution of the ionizing parameter throughout the system, the grids from the photoionization model MAPPINGS $\mathrm{V}^{5}$ (Sutherland, in prep.) are used on a $[\mathrm{OIII}] /[\mathrm{SII}]$ versus $[\mathrm{NII}] /[\mathrm{SII}]$ diagram, which offers a convenient way to break the degeneracy between

5 Available at miocene.anu.edu.au/Mappings 

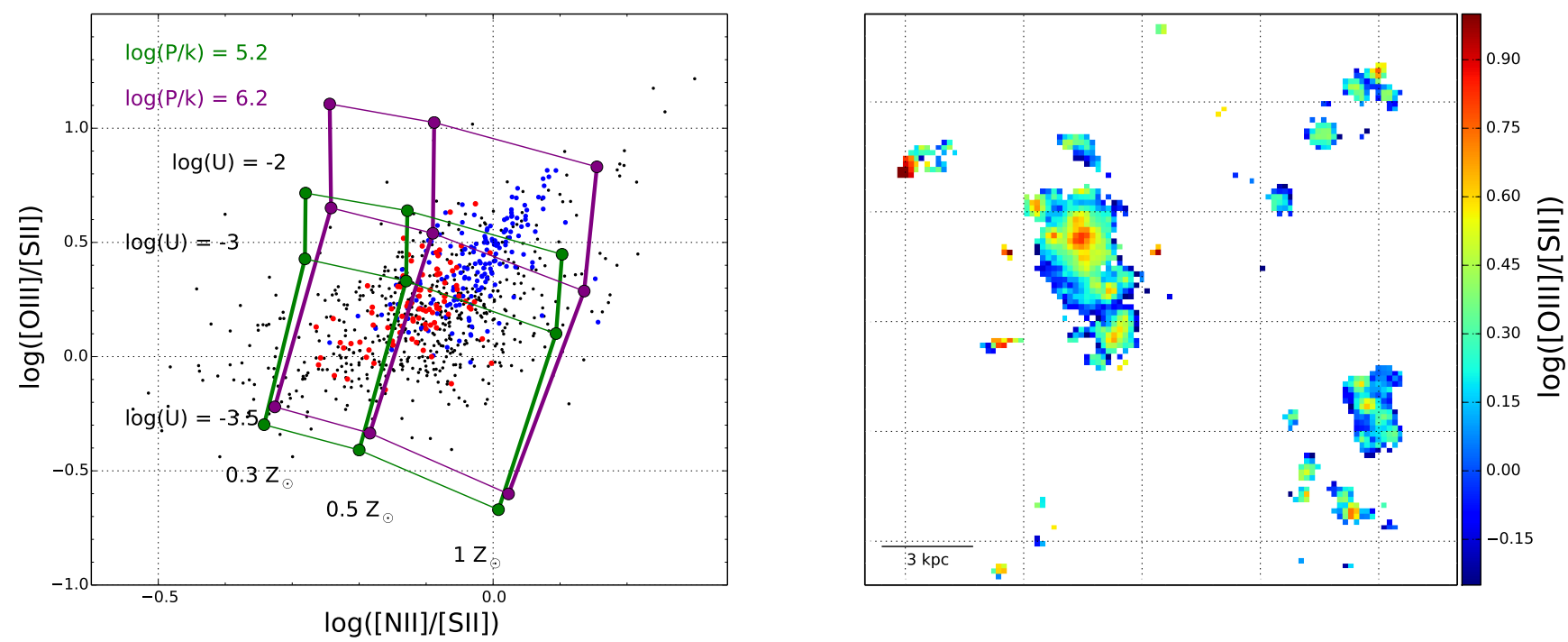

Fig. 10. Left: metallicity-ionization parameter grid from the MAPPINGS $V$ ionization model. The red points are the ones falling outside of the SF region in the third BPT diagram. The thick lines represent the grid for a constant metallicity and the thin lines for a constant ionizing parameter. The dimensionless ionizing parameter goes from $\log (U)=-3.5$ to $\log (U)=-2$. Right: spatial distribution of the logarithmic values of the [OIII]/[SII] ratio, used as a proxy for the ionization parameter. We see an outside-in gradient showing that the most ionized regions are located in the centre of the galaxy. The field of view is the same as in Fig. 3.

the metallicity and ionizing parameters. The electronic density, computed from the $[\mathrm{SII}]_{\lambda 6717} /[\mathrm{SII}]_{\lambda 6731}$ ratio, reaches a maximum value of $300 \mathrm{~cm}^{-3}$ (see Sect. 2.1) and can get as low as $9 \mathrm{~cm}^{-3}$ (see Sect. 4.1.2). Moreover, the electronic temperature was estimated to be $12700 \mathrm{~K}$ by DM98. Two grids were then used, with $\log (P / k)=5.2$ and 6.2 to cover the density range encountered in the galaxy. The shape of the ionizing extreme ultraviolet photon spectrum comes from the STARBURST99 synthesis model (Leitherer et al. 1999), assuming a Saltpeter IMF, Lejeune et al. (1997) atmospheres, and a continuous starformation extending over $4 \mathrm{Myr}$, as described in Dopita et al. (2013). The left-hand panel of Fig. 10 shows that the ionizing parameter values are quite dispersed, going from $\log (U)=-3.5$ to $\log (U)=-2$. The spatial distribution of the [OIII $]$ [ [SII] emission line ratio, which can be used as a proxy for the ionization parameter, is plotted in the right-hand panel of Fig. 10, shows a similar gradient to the one observed in Fig. 5 , and can therefore be accounted for the spread observed in the three BPT diagrams.

\subsection{Continuum emission}

Besides the emission lines, the continuum emission from the MUSE spectra was also extracted, and its spatial distribution investigated. Away from the main star-forming regions, this continuum emission is quite faint and hardly above the noise level. As shown in Figs. 4 and 11, this diffuse emission is spatially extended and rather blue $(V-R \simeq 0.2 \mathrm{mag})$. The existence of this diffuse component is secured by the deep broad band images obtained with FORS: see the similarity between the reconstructed MUSE map of Fig. 11 and the composite image of FORS shown in the upper right-hand panel of Fig. 2. Figure 12 further shows that this component extends into areas where no strong emission lines are observed, especially not $\mathrm{H} \alpha$.

\section{Discussion}

The target investigated here, NGC $5291 \mathrm{~N}$, is supposedly a "simple" object. It was formed less than a few hundred Myr ago

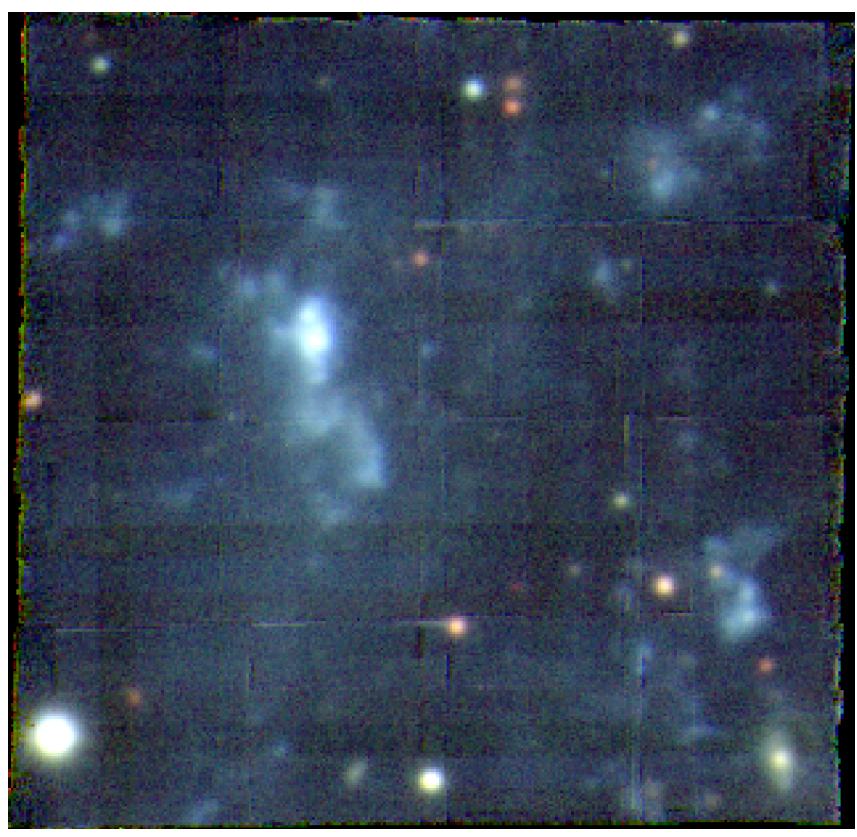

Fig. 11. Reconstructed composite colour image of the extracted continuum of NGC $5291 \mathrm{~N}$ using similar bands to $V, R$, and $I$ on MUSE. The blue diffuse continuum emission is detected, although less clearly than in the FORS image presented in the upper right panel of Fig. 2. The image was created from the unbinned data cube.

following a high speed collision in an initially purely gaseous structure (Bournaud et al. 2007). Nevertheless, the FORS deep images and MUSE data presented here already show a complex ISM. The ISM in this still gas-dominated object appears to be very clumpy, like the typical star-forming galaxies at $z>1$. The high spatial resolution and large field of view of MUSE allowed us to map the flux distribution of multiple emission lines, as well as the flux ratios between them up to large radial distances.

Large and small scale variations in emission line ratios are observed. In particular, in some regions the $[\mathrm{OI}] / \mathrm{H} \alpha$ ratio is 


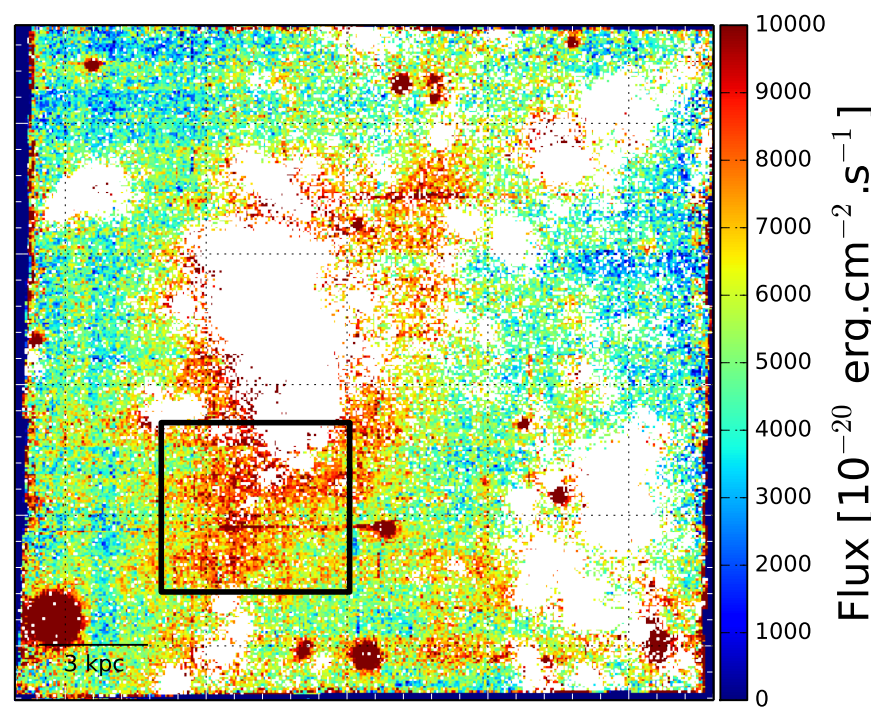

Fig. 12. Integrated emission of the continuum in $10^{-20} \mathrm{erg} \mathrm{s}^{-1} \mathrm{~cm}^{2}$ between $4750 \AA$ and $6000 \AA$. Spaxels for which a $3 \sigma \mathrm{H} \alpha$ emission was detected are shown in white. The black rectangle indicates the region whose spectra were combined to obtain the stacked spectra of Fig. 15. The field of view is the same as in Fig. 3.

found beyond the locus of typical starbursts. Deviant points surround the main star-forming regions and are thus found at relatively low $\mathrm{H} \alpha$ surface brightness. Such variations in the emission lines cannot be due to local changes in the dust extinction or metallicity, which we found to be pretty uniform. We discuss here other possible origins for the deviant line ratios.

\subsection{Origin of the strong $[\mathrm{OI}] / \mathrm{H} \alpha$ emission}

The $[\mathrm{OI}] \lambda 6300$ is a faint line, making it difficult to map. However, the sensitivity of MUSE allowed us to detect it with a $S / N>3$ in extended regions.

The stacked spectrum (see error bars in Fig. 7) unambiguously confirms the high level of [OI] emission outside the main $\mathrm{H}$ II regions. Thus, the high $[\mathrm{OI}] / \mathrm{H} \alpha$ line ratios are unlikely to be due to measurement errors.

\subsubsection{Low-density diffuse ionized gas}

The regions with strong $[\mathrm{OI}] / \mathrm{H} \alpha$ ratios surround the main starforming knots and lie within the warm low-density ionized phase of the ISM, often referred to as the diffuse ionized gas (DIG). The DIG is ionized by field OB stars, leaking photons from H II regions (Hoopes \& Walterbos 2003), or by shocks. The low density of the DIG is known to harden the ionizing spectra and can lead to LINER-like line ratios. However, a very low ionization parameter, below $\log (U)<-4$ (see Hoopes \& Walterbos 2003 ), is required to reach the observed values of the $[\mathrm{OI}] / \mathrm{H} \alpha$ line ratios. As shown in Sect. 3.5 , the data points on the $[\mathrm{OI}] / \mathrm{H} \alpha$ diagram constrain the ionization parameter to be in the range $-3.5<\log (U)<-2$. This is much higher than the value needed to get non-stellar ionization-like line ratios in the DIG.

\subsubsection{Shocks}

Shocks due to stellar winds, supernovae explosions, or dynamical processes, such as collisions or accretion of gas, can also produce large forbidden-to-Balmer line ratios. We tested the shock hypothesis with the fast radiative shock model from Allen et al. (2008), which is based on MAPPINGS III (Sutherland \& Dopita 1993).

The shock model library has a limited number of input parameters. We have assumed an electronic density of $1 \mathrm{~cm}^{-3}$ and an LMC abundance, because these parameters are the closest available to those estimated in the ISM of NGC 5291N. The speed of the shock and the transverse magnetic parameter were varied, from 100 to $300 \mathrm{~km} \mathrm{~s}^{-1}$ and from 0 to $10 \mu \mathrm{G}$, respectively.

The locus of the resulting model is shown in Fig. 13 for shock only and shock + precursors. We find that the outlying data points are encompassed between the grids for shock and shock + precursors. For comparison, the prediction of pure stellar photo-ionization from MAPPINGS V is also shown in Fig. 13. Because the average electronic density of the points lying outside of the "star-forming" region in the third BPT diagram is around $10 \mathrm{~cm}^{-3}$, we used the model with $\log (P / k)=5.2$ (see Sect. 3.5). A half-solar metallicity was also assumed.

The velocity dispersion map of the ISM was computed from the width of the $\mathrm{H} \alpha$ emission line. We subtracted a constant value of $2.34 \AA$ from the width of the $\mathrm{H}_{\alpha}$ line, corresponding to the average Gaussian FWHM close to the $\mathrm{H} \alpha$ line. The map, shown in Fig. 14, has peaks at $\sigma>130 \mathrm{~km} \mathrm{~s}^{-1}$ in the central regions - most likely due to spatially unresolved rotation (Bournaud et al. 2004) - but also in the outskirts of the main star-forming knots close to the region where $[\mathrm{OI}] / \mathrm{H} \alpha$ culminates. A detailed inspection reveals that the high- $\sigma$ regions are in fact located a bit farther away where the [OI] emission is below the detection limit. However, the observed line-of-sight velocity dispersions could be consistent with transverse shocks up to $300 \mathrm{~km} \mathrm{~s}^{-1}$. If shocks are responsible for the high [OI]/H $\alpha$ ratios, what is their origin?

First, a shock could have arisen from the accretion of gas onto the newly formed dwarf. In this case we would expect the shocks to only be present around the galaxy because the accretion of gas is supposed to occur towards the deepest gravitational potential well, hence all around the dwarf galaxy. However, as one can see in Fig. 7, the strong [OI]/H $\alpha$ ratio regions are not only found around the kinematically decoupled dwarf galaxy but also around most star-forming regions away from NGC 5291N (See the south-west clump, for instance).

Second, a shock could be due to the stellar feedback from the very recent starburst. Strong outflows in the centre of the star-forming region would then be expected. The expected kinematical signature of such outflows - an additional broad component in the emission lines - is not seen in the MUSE data: single Gaussian fits properly fit the $\mathrm{H} \alpha$ emission line, even in the centre of the star-forming regions ${ }^{6}$.

Third, the high $\sigma$ velocities could be the remaining signature of the violent collision between NGC 5291 and a massive interloper that created the HI ring. The shock signature could have been wiped out in the central star-forming region because of the stellar activity and only remain in the low-density gas surrounding this area. In the simulation of Bournaud et al. (2007) of the ring formation and expansion, the induced velocity dispersion 0.7 Gyr after the collision had decreased to about $\sigma \simeq 40 \mathrm{~km} \mathrm{~s}^{-1}$.

6 Fabry-Perot $\mathrm{H} \alpha$ data with high spectral resolution for this region are available (Bournaud et al. 2004). Their spectral resolution $(R=9375)$ are far superior to the MUSE data $(R=2840$ close to $\mathrm{H} \alpha)$. Their analysis reveal complex kinematical features, with likely superimposed foreground and background clouds, but no obvious signature of outflows. The broad wings of the $\mathrm{H} \alpha$ line in their data are instrumental signatures. 

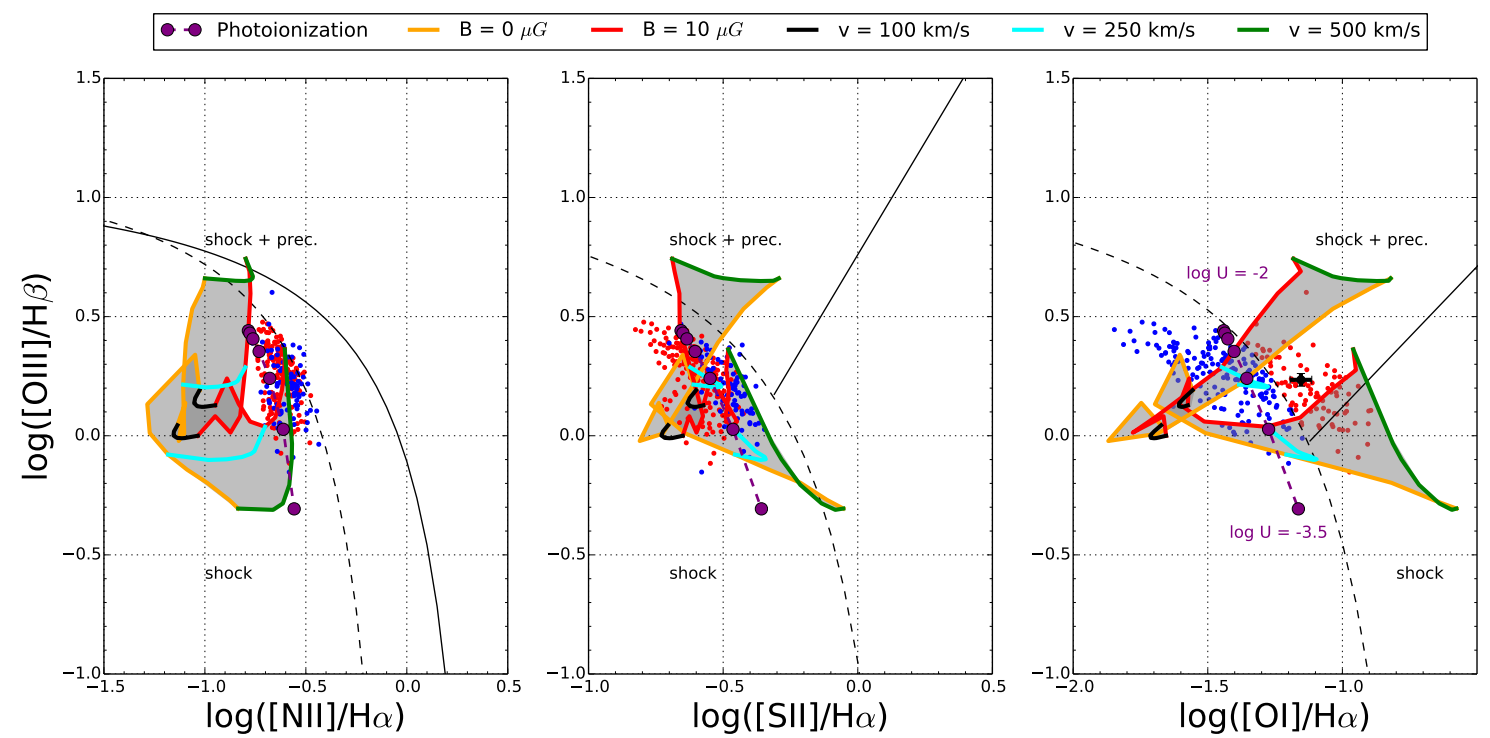

Fig. 13. Shock models on the BPT diagrams. Only points with detected [OI] emission are plotted. The blue (resp. red) points lie in (resp. out of) the star formation locus on the $[\mathrm{OI}] / \mathrm{H} \alpha$ BPT diagram. The purple dashed line is the ionization model prediction from MAPPINGS V, using a metallicity $Z=0.5 Z_{\odot}$ and $\log (P / k)=5.2$. The ionization parameter goes from $\log U=-2$ to $\log U=-3.5$, as indicated in the third diagram. The two grids come from the MAPPINGS III model for fast shock, as described in the text, and are drawn for shock only and shock + precursor.

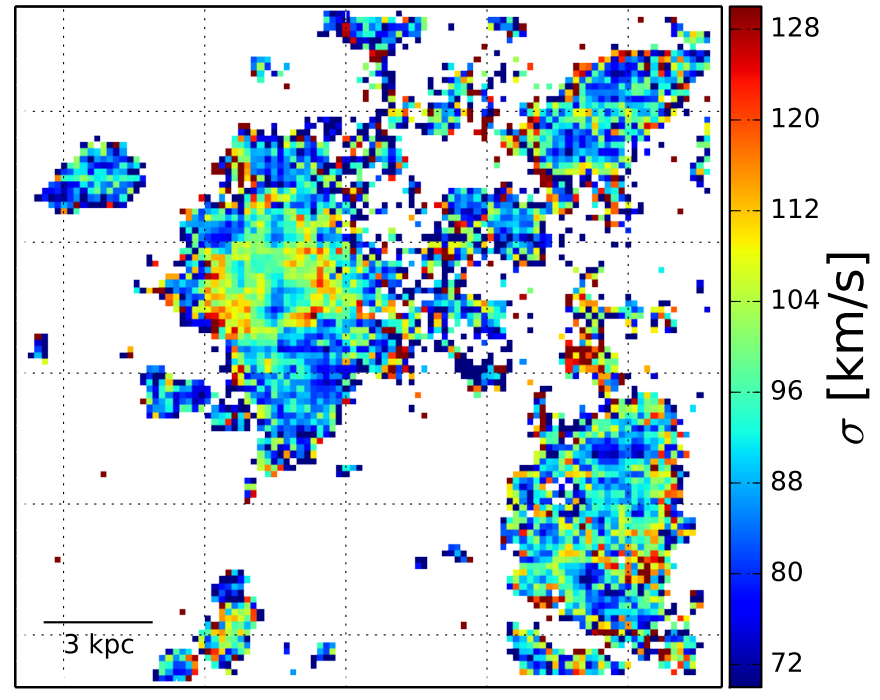

Fig. 14. Velocity dispersion measured from the width of the $\mathrm{H} \alpha$ emission line. The field of view is the same as in Fig. 3.

According to the shock models, this is too low to account for the high $[\mathrm{OI}] / \mathrm{H} \alpha$ flux ratio.

The models involving a photoionization within a very lowdensity environment (the DIG hypothesis) or shocks therefore do not provide satisfactory explanations for the values and spatial variations of the $[\mathrm{OI}] / \mathrm{H} \alpha$ flux ratios. Confirming the existence of regions with high $[\mathrm{OI}] / \mathrm{H} \alpha$ in the clumpy ISM of collisional debris (or of distant galaxies) should be a first step in our understanding of the ionization process. In fact, Cairós et al. (2010), who obtained IFU data for eight dwarf galaxies considered as BCDGs with the Postdam Multi-Aperture Spectrophotometer (PMAS) at the $3.5 \mathrm{~m}$ telescope at Calar Alto Observatory, also measured high values of $[\mathrm{OI}] / \mathrm{H} \alpha-$ as high as $\log ([\mathrm{OI}] / \mathrm{H} \alpha)=$ -0.50 - for several objects in their sample. It is remarkable that these high ratios are always located in the outskirts of the galaxies. In some of them, I Zw 159 and Mrk 32, they also suggest the presence of shocks in the same regions, based on strong values of the $[\mathrm{SII}] / \mathrm{H} \alpha$ ratio, above $\log ([\mathrm{SII}] / \mathrm{H} \alpha)=-0.20$. However the lack of spatial resolution prevents firm conclusions. Strong outflows presumably generating shocks have been found in ESO338-IG04, the only other dwarf galaxy for which MUSE data are available (Bik et al. 2015). Outflows were disclosed in the analysis of the $\mathrm{H} \alpha$ kinematic maps, and highly ionized cones were probed by using the $[\mathrm{SII}] /[\mathrm{OIII}]$ line ratio, thought to be escape gates to Ly $\alpha$ photons. It is interesting to note that no such feature was observed in NGC 5291N.

\subsection{Origin of the diffuse continuum emission}

As presented in Sect. 3.6, the $\mathrm{H} \alpha$-emitting regions are surrounded by a diffuse blue light emission of still unknown origin.One may speculate that this light has been emitted by a stellar population old enough to no longer ionize the surrounding gas. This would be consistent with the outside-in evidence of the star-formation events derived from the $\mathrm{H} \beta$ equivalent width map. To characterize the physical properties of this diffuse component further, we have stacked the spectra over the full south-eastern region where the diffuse continuum emission is most prominent. The resulting spectrum does not reveal any underlying absorption line (see Fig. 15) or actually residual emission lines. This puts very strong constraints on the age of the putative underlying stellar population; galaxy SED models such as GALEV (Kotulla et al. 2009) show that the transition between emission and the absorption line for $\mathrm{H} \beta$ occurs at around $25 \mathrm{Myr}$ after an instantaneous burst and does not last more than a few Myr. One would then witness a special episode of the star formation history: the transition between the death of the OB stars and emergence of type-A absorption lines, lasting a very short time but occurring in an extended region, spanning more than $3 \mathrm{kpc}$. This would mean that the star-formation episode occurred almost simultaneously on a $3 \mathrm{kpc}$ scale region, which is rather unrealistic.

A second hypothesis would be that this emission is actually scattered light from the star-forming region. In this case, one should expect to see an associated UV counterpart, that is 
J. Fensch et al.: ISM properties of a local analogue to high redshift star-forming clumps

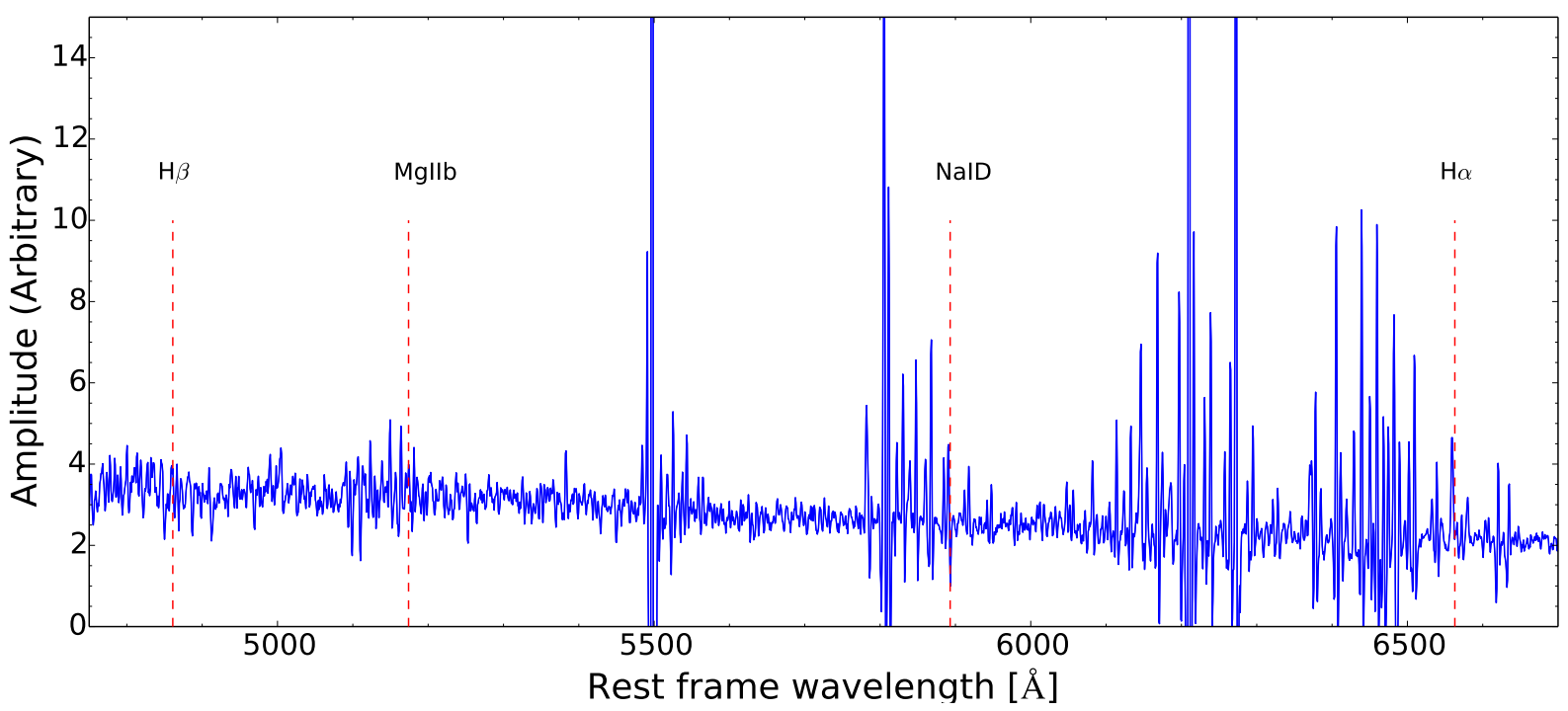

Fig. 15. Stacked spectra from the south-eastern region indicated by a black rectangle in Fig. 12. Every spaxel showing a $3 \sigma$ H $\alpha$ detection was rejected.

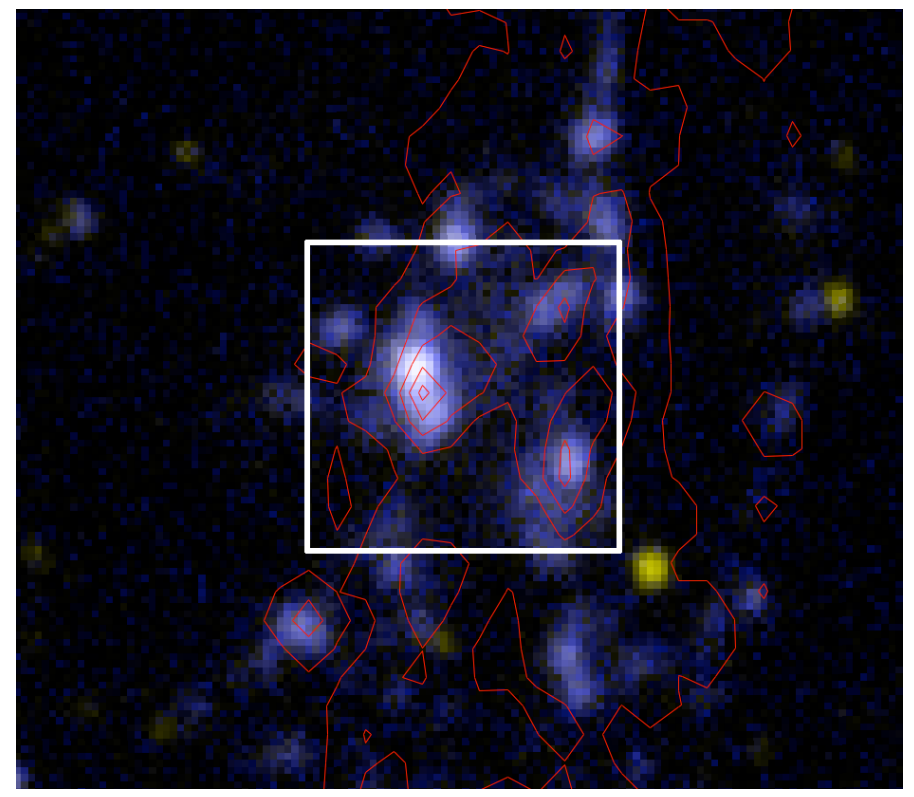

Fig. 16. Composite colour image of the UV emission as seen by GALEX. The near-UV is shown in red and green and the far-UV in blue. HI contours are superimposed in red. The MUSE field of view is shown by the white square.

dust scattered leaking UV emission from the hot young stars (see Boissier et al. 2015, and references therein). However, the GALEX data (see description in Boquien et al. 2007, 2009) of this system showed that there is no near-UV nor far-UV emission towards the optical blue diffuse component (compare Figs. 12 and 16). One should note that the GALEX data are quite deep with an exposition time of about $9000 \mathrm{~s}$ for the NUV and $5500 \mathrm{~s}$ in FUV.

Thus, the origin of this diffuse component remains undetermined. MUSE observations of other similar systems (or of other parts of the NGC 5291 gaseous ring) should tell how common it is around starbursting galaxies.

\section{Conclusions}

We have presented FORS deep multi-band optical images and high spatial resolution IFU data on NGC $5291 \mathrm{~N}$, one of the very few starbursting dwarfs yet observed with the revolutionary instrument MUSE on the ESO VLT. This dwarf, located at $63 \mathrm{Mpc}$ and formed within a ring of gas expelled from the host galaxy, NGC 5291, after a violent collision several $10^{8}$ years ago. NGC 5291N shares a number of physical properties with clumps in gas-rich star-forming high-redshift galaxies: it is very gaseous, has a clumpy ISM with sub-solar metallicity and contains no old stars, unlike classical blue compact dwarf galaxies.

The MUSE data cube provided us with optical spectra in the range 4750-9350 $\AA$ over a field of view of about $1^{\prime} \times 1^{\prime}$. Each spaxel corresponds to $60 \mathrm{pc} \times 60 \mathrm{pc}$ at the distance of NGC $5291 \mathrm{~N}$, before rebinning the data cube. Strong emission lines $(\mathrm{H} \beta,[\mathrm{OIII}],[\mathrm{OI}], \mathrm{H} \alpha,[\mathrm{NII}]$, and [SII]) and continuum emission were extracted and mapped to investigate the properties of the ISM. The derived maps showed a rather strong heterogeneity in the spatial distributions of the different emission lines and an extended blue diffuse continuum emission.

Emission line ratios were investigated with BPT-like diagnostic diagrams. They showed that most data point are consistent with star-forming regions, with a noticeably large scatter on the diagrams. Furthermore, a significant number of points fall outside of the star-formation locus of the $[\mathrm{OIII}] / \mathrm{H} \beta$ vs. [OI] $/ \mathrm{H} \alpha$ diagnostic diagram. They correspond to regions located on the outskirts of the dwarf galaxy.

The equivalent width of the $\mathrm{H} \beta(\mathrm{EW}(\mathrm{H} \beta))$ line shows that the galaxy has undergone a very recent starburst: no older than $3 \mathrm{Myr}$ in the brightest region to $7 \mathrm{Myr}$ old in the fainter neighbourhood under the hypothesis of an instantaneous burst. The observed distribution of $\mathrm{EW}(\mathrm{H} \beta)$ is consistent with an outsidein star formation episode. The extinction map $\left(A_{v}\right)$ computed from the Balmer line ratio $\mathrm{H} \alpha / \mathrm{H} \beta$ indicates that the dust concentrates towards the most active star-forming regions, where mid- and far-infrared emission had already been found. This is also the region where the $V-R$ colour map derived from the FORS broad-band images shows narrow red filaments that are thus most likely associated with dust lanes.

The metallicity was computed using the empirical O3N2 calibration and gave a mean value $\langle 12+\log (\mathrm{O} / \mathrm{H})\rangle=8.4$ with a standard deviation of 0.05 (equivalent to an abundance of $Z \simeq 0.5 Z_{\odot}$ ), confirming previous estimates from slit spectroscopy. This relatively high value is fully expected for a recycled dwarf galaxy made in collisional debris. A surprising 
inside-out positive metallicity gradient is observed; it is at odds with predictions from numerical simulations of the chemical evolution of tidal dwarf galaxies. However, the metallicity distribution spreads over less than $0.2 \mathrm{dex}$, which is the intrinsic uncertainty of the empirical method. The computed O3N2 map is therefore consistent with a homogeneous metallicity coupled with variations in other physical parameters, such as the ionization parameter.

The spatial distribution of the ionization parameter was determined through a $[\mathrm{OIII}] /[\mathrm{SII}]$ versus $[\mathrm{NII}] /[\mathrm{SII}]$ photoionization grid computed with the MAPPINGS V model. The inferred ionization parameter spreads over a wide range of values from $\log (U)=-3.5$ to $\log (U)>-2$, and this explains the observed scatter in the BPT diagrams, except for the points with high $[\mathrm{OI}] / \mathrm{H} \alpha$ flux ratios which are not compatible with simple stellar photoionization models. Models involving the low-density DIG, which is hot and only partially ionized, can emit non-stellar emission line ratios, but they need a ionization parameter lower than the one derived from the MUSE data.

Various shock models were investigated. They can reproduce the observed flux ratios that are not compatible with stellar photoionization for shock velocities higher than $200 \mathrm{~km} \mathrm{~s}^{-1}$. The velocity dispersion map computed from the IFU data cube do show an increase in $\sigma(V)$ outside the main star-forming regions, but at a level and precise location that are not fully consistent with the shock model.

An extended blue diffuse emission surrounding the main star-forming regions is observed in the deep optical FORS images and detected with MUSE. The IFU data indicates that this is pure continuum emission, exhibiting neither emission nor absorption lines, expanding over distances of at least $3 \mathrm{kpc}$ from the emission line regions. The hypothesis of emission arising from a stellar population old enough not to ionize the surrounding HI gas, but also young enough not to exhibit Balmer absorption lines, appears rather unlikely. Besides this, the UV emission associated with scattered light from the central star formation region was not observed by the GALEX UV observatory.

No firm conclusions could therefore be reached about the origin of the deviant points in the $[\mathrm{OI}] / \mathrm{H} \alpha$ BPT diagram, which is spatially located just outside the starburst regions of the dwarf, and of the pure diffuse blue optical continuum emission found farther out. This calls for further IFU observations of the outskirts of nearby star-forming galaxies, in particular the youngest ones formed in the debris of galaxy-galaxy collisions, which are arguably the best local proxies of the distant star-forming objects.

Acknowledgements. The authors thank Michael Dopita and Stephanie Juneau for very useful discussions, and the anonymous referee for in-depth reading and helpful comments. P.M.W. received funding through BMBF Verbundforschung (project MUSE-AO, grant 05A14BAC). E.Z. acknowledges funding from the Swedish Research Council (project 2011-5349). This research made use of the NASA/IPAC Extragalactic Database (NED) which is operated by the Jet Propulsion Laboratory, California Institute of Technology, under contract with the National Aeronautics and Space Administration.

\section{References}

Allen, M. G., Groves, B. A., Dopita, M. A., Sutherland, R. S., \& Kewley, L. J. 2008, ApJS, 178, 20

Asplund, M., Grevesse, N., Sauval, A. J., \& Scott, P. 2009, ARA\&A, 47, 481 Bacon, R., Accardo, M., Adjali, L., et al. 2010, in SPIE Conf. Ser., 7735, 8
Baldwin, J. A., Phillips, M. M., \& Terlevich, R. 1981, PASP, 93, 5

Bik, A., Östlin, G., Hayes, M., et al. 2015, A\&A, 576, L13

Boissier, S., Boselli, A., Voyer, E., et al. 2015, A\&A, 579, A29

Boquien, M., Duc, P.-A., Braine, J., et al. 2007, A\&A, 467, 93

Boquien, M., Duc, P.-A., Wu, Y., et al. 2009, AJ, 137, 4561

Boquien, M., Duc, P.-A., Galliano, F., et al. 2010, AJ, 140, 2124

Bournaud, F., Duc, P.-A., Amram, P., Combes, F., \& Gach, J.-L. 2004, A\&A, 425,813

Bournaud, F., Duc, P.-A., Brinks, E., et al. 2007, Science, 316, 1166

Bournaud, F., Perret, V., Renaud, F., et al. 2014, ApJ, 780, 57

Cairós, L. M., Caon, N., Papaderos, P., et al. 2009, ApJ, 707, 1676

Cairós, L. M., Caon, N., Zurita, C., et al. 2010, A\&A, 520, A90

Dopita, M. A., Sutherland, R. S., Nicholls, D. C., Kewley, L. J., \& Vogt, F. P. A. 2013, ApJS, 208, 10

Duc, P.-A., \& Mirabel, I. F. 1998, A\&A, 333, 813

Duc, P.-A., \& Mirabel, I. F. 1999, in Galaxy Interactions at Low and High Redshift, eds. J. E. Barnes, \& D. B. Sanders, IAU Symp., 186, 61

Erb, D. K., Shapley, A. E., Pettini, M., et al. 2006, ApJ, 644, 813

Fioc, M., \& Rocca-Volmerange, B. 1997, A\&A, 326, 950

Fioc, M., \& Rocca-Volmerange, B. 1999, ArXiv e-prints [arXiv: astro-ph/9912179]

Genel, S., Naab, T., Genzel, R., et al. 2012, ApJ, 745, 11

Gordon, K. D., Clayton, G. C., Misselt, K. A., Landolt, A. U., \& Wolff, M. J. 2003, ApJ, 594, 279

Hoopes, C. G., \& Walterbos, R. A. M. 2003, ApJ, 586, 902

Izotov, Y. I., Schaerer, D., Blecha, A., et al. 2006, A\&A, 459, 71

James, B. L., Tsamis, Y. G., Barlow, M. J., et al. 2009, MNRAS, 398, 2

James, B. L., Tsamis, Y. G., \& Barlow, M. J. 2010, MNRAS, 401, 759

James, B. L., Tsamis, Y. G., Barlow, M. J., Walsh, J. R., \& Westmoquette, M. S. 2013a, MNRAS, 428, 86

James, B. L., Tsamis, Y. G., Walsh, J. R., Barlow, M. J., \& Westmoquette, M. S. 2013b, MNRAS, 430, 2097

Kauffmann, G., White, S. D. M., \& Guiderdoni, B. 1993, MNRAS, 264, 201

Kauffmann, G., Heckman, T. M., Tremonti, C., et al. 2003, MNRAS, 346, 1055

Kehrig, C., Pérez-Montero, E., Vílchez, J. M., et al. 2013, MNRAS, 432, 2731

Kewley, L. J., Dopita, M. A., Sutherland, R. S., Heisler, C. A., \& Trevena, J. 2001, ApJ, 556, 121

Kewley, L. J., Groves, B., Kauffmann, G., \& Heckman, T. 2006, MNRAS, 372, 961

Kotulla, R., Fritze, U., Weilbacher, P., \& Anders, P. 2009, MNRAS, 396, 462

Kunth, D., Maurogordato, S., \& Vigroux, L. 1988, A\&A, 204, 10

Lagos, P., Telles, E., Muñoz-Tuñón, C., et al. 2009, AJ, 137, 5068

Lagos, P., Telles, E., Nigoche Netro, A., \& Carrasco, E. R. 2012, MNRAS, 427, 740

Lagos, P., Papaderos, P., Gomes, J. M., Smith Castelli, A. V., \& Vega, L. R. 2014, A\&A, 569, A110

Leitherer, C., Schaerer, D., Goldader, J. D., et al. 1999, ApJS, 123, 3

Lejeune, T., Cuisinier, F., \& Buser, R. 1997, A\&AS, 125, 229

Lelli, F., Duc, P.-A., Brinks, E., et al. 2015, A\&A, 584, A113

Loose, H.-H., \& Thuan, T. X. 1986, in Star-forming Dwarf Galaxies and Related Objects, eds. D. Kunth, T. X. Thuan, J. Tran Thanh Van, J. Lequeux, \& J. Audouze, 73

Marino, R. A., Rosales-Ortega, F. F., Sánchez, S. F., et al. 2013, A\&A, 559, A114 Maza, J., Ruiz, M. T., Gonzalez, L. E., Wischnjewsky, M., \& Pena, M. 1991, A\&AS, 89, 389

Osterbrock, D. E., \& Bochkarev, N. G. 1989, Sov. Astron., 33, 694

Papaderos, P., Loose, H.-H., Thuan, T. X., \& Fricke, K. J. 1996, A\&AS, 120, 207

Pettini, M., \& Pagel, B. E. J. 2004, MNRAS, 348, L59

Ploeckinger, S., Hensler, G., Recchi, S., Mitchell, N., \& Kroupa, P. 2014, MNRAS, 437, 3980

Sargent, W. L. W., \& Searle, L. 1970, ApJ, 162, L155

Stark, D. P., Swinbank, A. M., Ellis, R. S., et al. 2008, Nature, 455, 775

Stasińska, G., \& Leitherer, C. 1996, ApJS, 107, 661

Steidel, C. C., Rudie, G. C., Strom, A. L., et al. 2014, ApJ, 795, 165

Sutherland, R. S., \& Dopita, M. A. 1993, ApJS, 88, 253

Terlevich, R., Silich, S., Rosa-González, D., \& Terlevich, E. 2004, MNRAS, 348, 1191

Veilleux, S., \& Osterbrock, D. E. 1987, ApJS, 63, 295

Weilbacher, P. M., Streicher, O., Urrutia, T., et al. 2012, in Proc. SPIE, 8451

Zackrisson, E., Bergvall, N., Olofsson, K., \& Siebert, A. 2001, A\&A, 375, 814

Zanella, A., Daddi, E., Le Floc'h, E., et al. 2015, Nature, 521, 54 